Pure and Applied Mathematics Quarterly

Volume 8, Number 4

$941-955,2012$

\title{
Non-simply Laced McKay Correspondence and Triality
}

\author{
Naichung Conan Leung and Jiajin Zhang
}

\begin{abstract}
The classical McKay correspondence establishes a one-to-one correspondence between finite subgroups of $S U(2)$ and simply-laced root systems, namely root systems of $A D E$ type. In this article, we extend the McKay correspondence to all root systems, simply-laced or not, and relate this correspondence to triality of quaternions.
\end{abstract}

Keywords: McKay correspondence, quaternion, triality, crepant resolution.

\section{INTRODUCTION}

1.1. The McKay correspondence says that, conjugacy classes of finite subgroups $\widetilde{\Gamma}$ of $S U(2)$, are in one-to-one correspondence with simply-laced simple Lie algebras, or equivalently, Dynkin diagrams of $A D E$ type.

In this article, we extend this correspondence to all simple Lie algebras, simplylaced or not, using the triality of the quaternions $\mathbb{H}$. This correspondence for non-simply laced Dynkin diagrams is more or less known. We give an explicit and unified description, and relate it to the triality of the quaternions $\mathbb{H}$.

Note that $S U(2)=S p(1)$ acts on $\mathbb{H}$ by left multiplications and the adjoint map $A d: S U(2) \rightarrow S O(3)$ is the universal covering of $S O(3)$, which is the automorphism group of $\mathbb{H}$ as a normed division algebra, i.e. $S O(3)=A u t(\mathbb{H})$ and $S U(2)=\widehat{A u t}(\mathbb{H})$. Recall that there are only four normed division algebras $\mathbb{A}$ and they are $\mathbb{R}, \mathbb{C}, \mathbb{H}$ and $\mathbb{O}$. Such an algebraic structure is closely related to the notion of a normed triality:

$$
t: V_{1} \times V_{2} \times V_{3} \rightarrow \mathbb{R}
$$

Indeed given any triality triple $\vec{v}=\left(v_{1}, v_{2}, v_{3}\right)$, we obtain canonical identifications $V_{1} \cong V_{2} \cong V_{3} \cong V_{3}^{*}$ and $t$ determines a product structure on $V_{i}$. This makes $V_{i}$

Received February 25, 2011

MSC 2010: Primary 14E16; Secondary 16H05. 
into a normed division algebra $\mathbb{A}$. We write $t_{\mathbb{A}}$ instead of $t$ and we denote the set of all triality triples as $\operatorname{Tr} i_{\mathbb{A}}$.

The symmetry group $A u t\left(t_{\mathbb{A}}\right) \subset O\left(V_{1}\right) \times O\left(V_{2}\right) \times O\left(V_{3}\right)$ of triality is isomorphic to $S U(2)^{3} / \mathbb{Z}_{2}$ (resp. Spin $(8)$ ) when $\mathbb{A}$ equals $\mathbb{H}$ (resp. $\mathbb{O}$ ). Also we denote the universal cover of $A u t\left(t_{\mathbb{A}}\right)$ by $\widetilde{A u t}\left(t_{\mathbb{A}}\right)$. Then $\widetilde{A u t}\left(t_{\mathbb{H}}\right)=S U(2)^{3}$. The projection to each $V_{i}$ induces a homomorphism $p_{i}: \widetilde{\operatorname{Aut}}\left(t_{\mathbb{A}}\right) \rightarrow O\left(V_{i}\right)$. In general, Aut $\left(t_{\mathbb{A}}\right)$ acts transitively on $T r i_{\mathbb{A}}$ and the isotropy group is isomorphic to $\operatorname{Aut}(\mathbb{A})$, which equals $S O(3)$ (resp. $G_{2}$ ) when $\mathbb{A}$ equals $\mathbb{H}$ (resp. $\mathbb{O}$ ). As a result, subgroups of $\operatorname{Aut}(\mathbb{A})$ correspond to subgroups of $\operatorname{Aut}\left(t_{\mathbb{A}}\right)$ which fix some point in $\operatorname{Tr} i_{\mathbb{A}}$.

From the above discussions, the McKay correspondence can be rephrased as a correspondence between simply laced root systems and finite subgroups of $\widetilde{A u t}\left(t_{\mathbb{H}}\right)$ fixing some element in $T r i_{\mathbb{H}}$, and we call such a subgroup an isotropic subgroup. Our main result says that if we consider pairs of finite isotropic subgroups of $\widetilde{A u t}\left(t_{\mathbb{H}}\right)$ inducing the same symmetries on each $V_{i}$, then the McKay correspondence can be extended to all root systems.

A pair $(\mathfrak{g}, \tau)$ with $\mathfrak{g}$ a (complex) simple Lie algebra and $\tau$ an outer automorphism determines a Lie algebra $\mathfrak{g}^{\tau}$ by taking the fixed part. Equivalently, a simply laced Dynkin diagram $D$ and a diagram automorphism $\tau$ determines a Dynkin diagram $D_{\tau}$ by taking the folding. All non-simply laced simple Lie algebras (or equivalently, Dynkin diagrams) can be obtained from simply laced ones in such a way. Namely, $\mathfrak{g}^{\tau}=B_{n}, C_{n}, F_{4}, G_{2}$ when $\mathfrak{g}=D_{n+1}, A_{2 n-1}, E_{6}, D_{4}$ respectively with $\tau$ being of order 2 except for $D_{4}$, and 3 for $D_{4}$.

Theorem 1. There is a one-to-one correspondence between the pairs $(\mathfrak{g}, \tau)$ and the equivalence classes of pairs of finite isotropic subgroups $\widetilde{\Gamma}, \widetilde{\Gamma^{\prime}} \subset \widetilde{A u t}\left(t_{\mathbb{H}}\right)$ of the same order satisfying $p_{i}(\widetilde{\Gamma})=p_{i}\left(\widetilde{\Gamma}^{\prime}\right) \subset O\left(V_{i}\right) \cong O(4)$ for all $i$.

To prove this result, we first establish the following non-simply laced McKay correspondence. Our construction is different from the classical restricted-induced construction in [14] (see also [10]).

Theorem 2. The equivalence classes of pairs $\left(\widetilde{\Gamma}, O_{v}\right)$ with $\widetilde{\Gamma}$ a finite subgroup of $S U(2)$ and $O_{v}$ an element of the outer automorphism group of $\widetilde{\Gamma}$ induced by $A d(v)$ where $v \in \mathbb{H}$, are in one-to-one correspondence with the pairs $(\mathfrak{g}, \tau)$ as above. In particular, we obtain all non-simply laced root systems from the pairs $\left(\widetilde{\Gamma}, O_{v}\right)$.

The Dynkin diagram associated with a pair $\left(\widetilde{\Gamma}, O_{v}\right)$ is obtained as follows. Let $W_{0}, W_{1}, \cdots, W_{n}$ be all irreducible representations of $\left(\widetilde{\Gamma}, O_{v}\right) . W_{0}$ is the trivial one. Let $W$ be the standard representation of $S U(2)$. Assign a node $p_{l}$ to each $W_{l}$. Assume $W_{l} \otimes W \cong \oplus_{m} a_{l m} W_{m}$. When $a_{l m}=a_{m l} \neq 0$ (both are equal to 1 ), we draw $a_{l m}$ edges to connect the nodes $p_{l}$ and $p_{m}$. When $a_{l m} \neq a_{m l}$, one of them, 


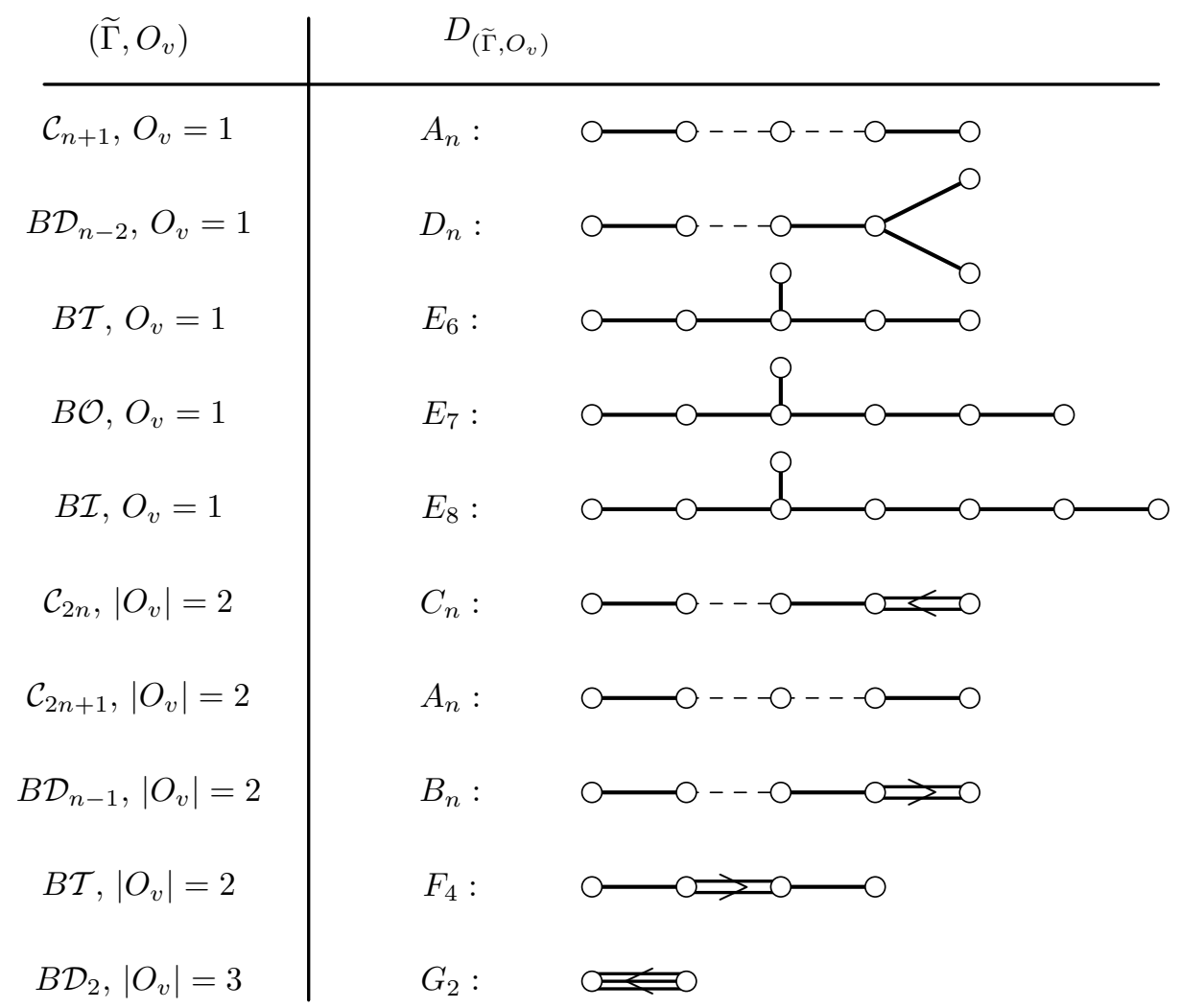

FiguRE 1. Dynkin diagrams associated with the pairs $\left(\widetilde{\Gamma}, O_{v}\right)$

say $a_{l m}$, must be 2 or 3 , and another is 1 . In this case, we draw $a_{l m}$ directed edges from $p_{m}$ to $p_{l}$. Thus, we obtain a diagram $D_{\left.\widetilde{\Gamma}, O_{v}\right)}^{a f f}$. Removing the node $p_{0}$ and all the edges with $p_{0}$ as an endpoint from $D_{\left(\widetilde{\Gamma}, O_{v}\right)}^{a f f}$, we obtain a diagram $D_{\left(\widetilde{\Gamma}, O_{v}\right)}$, and it is a Dynkin diagram. Conversely, each Dynkin diagram can be obtained in such a way. When $O_{v}=i d$, we obtain a simply laced Dynkin diagram. We illustrate this correspondence in Figure 1, where the groups $\mathcal{C}_{n}, B \mathcal{D}_{n}, B \mathcal{T}, B \mathcal{O}$, and $B \mathcal{I}$ are respectively the cyclic, binary dihedron, binary tetrahedron, binary octahedron, and binary icosahedron groups.

1.2. Remark. When $\mathbb{A}=\mathbb{R}$ the similar correspondence is rather trivial, as $\operatorname{Aut}(\mathbb{R})=\{1,-1\}$. When $\mathbb{A}=\mathbb{C}$ we have $U(1)=\operatorname{Aut}(\mathbb{C})$. Finite subgroups of $U(1)=S^{1}$ are finite cyclic groups $\mathbb{Z} / n \mathbb{Z}(n \in \mathbb{N})$. These finite groups are also subgroups of $S O(3)=\operatorname{Aut}(\mathbb{H})$, since $\operatorname{Aut}(\mathbb{C}) \subset \operatorname{Aut}(\mathbb{H})$.

When $\mathbb{A}=\mathbb{O}$, the octonions or the Cayley numbers, we have $\operatorname{Aut}(\mathbb{O})=G_{2}$ and $A u t\left(t_{\mathbb{O}}\right)=\operatorname{Spin}(8)$. Finite subgroups $\Gamma$ of $S U(3) \subset G_{2}$ were studied by 
many mathematicians, as well as physicists, for example, see [8][12][13][15][16] and the references therein, and the McKay correspondence relates the geometry of the Calabi-Yau threefolds which are the crepant resolutions of $\mathbb{C}^{3} / \Gamma$ and the orbifold (or stingy) geometry of $\mathbb{C}^{3} / \Gamma([12][13])$. There is also a version at the level of derived categories, see [3]. It is natural to ask a similar question for $\mathbb{R}^{7} / \Gamma$ and its $G_{2}$-resolution for finite subgroup $\Gamma \subseteq G_{2}$. It is also interesting to understand the octonions analogue of our result here.

1.3. In this short article, we first recall in $\S 2$ the classification of finite subgroups of $S U(2)$, and study their (special) outer automorphisms (i.e. automorphisms induced by adjoint actions).

In $\S 3$, we present an explicit description for non-simply laced McKay correspondence. Our result is Theorem 16 and Corollary 17 (see also Theorem 2).

In $\S 4$, we explain the relation between non-simply laced McKay correspondence and triality. The result is Theorem 1 (see also Theorem 25 and Corollary 26).

\section{Finite Subgroups of $S U(2)$}

2.1. Finite Subgroups of $S O(3)$ and $S U(2)$, and the Quaternions. Recall, finite subgroups of $S O(3)$ are classified completely, up to conjugacy (see [5][7]).

Proposition 3. The classification of finite subgroups $\Gamma$ of $S O(3)$, up to conjugacy, is listed in the following table. Here $A, B, C$ are the generators of $\Gamma$. The last column lists the regular polyhedrons whose symmetry groups are the respective finite groups.

\begin{tabular}{|c|c|c|c|}
\hline Group, $\Gamma$ & Order & Generating Relations & (Regular) Polyhedrons \\
\hline \hline Cyclic, $\mathcal{C}_{n}$ & $n$ & $A^{n}=B^{n}=C^{1}=A B C=1$ & $n$-gon's in $\mathbb{R}^{2}$ \\
\hline Dihedron, $\mathcal{D}_{n}$ & $2 n$ & $A^{n}=B^{2}=C^{2}=A B C=1$ & $n$-gon's in $\mathbb{R}^{3}$ \\
\hline Tetrahedron, $\mathcal{T}$ & 12 & $A^{3}=B^{3}=C^{2}=A B C=1$ & Tetrahedrons \\
\hline Octahedron, $\mathcal{O}$ & 24 & $A^{4}=B^{3}=C^{2}=A B C=1$ & Octahedrons \\
\hline Icosahedron, $\mathcal{I}$ & 60 & $A^{5}=B^{3}=C^{2}=A B C=1$ & Icosahedron \\
\hline
\end{tabular}

Let $\mathbb{H}$ be the algebra of quaternions, with the basis $1, \dot{1}, \dot{j}, \mathbb{k}$. For $a=a_{0}+$ $a_{1} \dot{\mathbb{1}}+a_{2, \dot{j}}+a_{3} \mathbb{k} \in \mathbb{H}$ with $a_{i} \in \mathbb{R}$, we denote $\operatorname{Re}(a)=a_{0} \in \operatorname{Re}(\mathbb{H}), \operatorname{Im}(a)=$ $a_{1} \dot{\mathbb{i}}+a_{2} \dot{j}+a_{3} \mathbb{k} \in \operatorname{Im}(\mathbb{H})$, and the conjugate $\bar{a}=\operatorname{Re}(a)-\operatorname{Im}(a)$. When $a_{0}=0$, $a$ is called a pure quaternion. If $a$ is a unit quaternion, then $a$ can be written as the form

$$
a=\cos \alpha+y \sin \alpha=e^{y \alpha},
$$

where $\cos \alpha=\operatorname{Re}(a)$ and $y$ is a pure unit quaternion. 
The set of all unit quaternions forms a multiplicative group, which is isomorphic to $S U(2)$ :

$$
a+b \dot{\mathbb{1}}+c \dot{\mathfrak{j}}+d \mathbb{k} \mapsto\left(\begin{array}{cc}
a+b \dot{\mathrm{i}} & c+d \dot{\mathrm{i}} \\
-c+d \dot{\mathrm{i}} & a-b \dot{\mathrm{i}}
\end{array}\right) .
$$

Identifying $\mathbb{H}$ with $\mathbb{C}^{2}$ by the map

$$
x=x_{0}+x_{1} \dot{\mathbb{1}}+x_{2} \dot{\mathfrak{j}}+x_{3} \mathbb{k} \mapsto\left(x_{0}+x_{1} \dot{\mathbb{1}},-x_{2}+x_{3} \dot{\mathrm{I}}\right),
$$

we see that $g(x)=g \cdot x$, for any $x \in \mathbb{H}, g \in S U(2)$.

In $\mathbb{R}^{3}=\operatorname{Im}(\mathbb{H})$, the reflections and rotations can be represented by quaternion multiplications.

Remark 4. (See [6]) Let y be a unit pure quaternion.

(1) Denote $S_{y}$ the reflection of $\operatorname{Im}(\mathbb{H})$ in the plane perpendicular to $y$. Then $S_{y}$ is represented by the quaternion transformation

$$
S_{y}(x)=y x y \text { for } x \in \operatorname{Im}(\mathbb{H}) .
$$

(2) Denote $R_{(y, \alpha)}$ the rotation of $\operatorname{Im}(\mathbb{H})$ about $y$ through $2 \alpha$. Then $R_{(y, \alpha)}$ is represented by the quaternion transformation

$$
R_{(y, \alpha)}(x)=e^{-\alpha y} x e^{\alpha y} \text { for } x \in \operatorname{Im}(\mathbb{H}) .
$$

(3) The group of all unit quaternions is $2: 1$ homomorphic to the group of all rotations that leave the origin fixed, that is, the group $S O(3)$. The kernel of this homomorphism is $\{ \pm 1\}$.

Let $\widetilde{\Gamma} \neq\{0\}$ be a finite subgroup of unit quaternions, or equivalently $\widetilde{\Gamma} \subset S U(2)$. There are two different classes.

The first class: If $\widetilde{\Gamma}$ contains -1 , then by Remark $4, \widetilde{\Gamma}$ is $2: 1$ homomorphic to a finite subgroup $\Gamma$ of $S O(3)$. By Proposition 3, there are 5 cases. Moreover, in terms of generators and generating relations, $\widetilde{\Gamma}$ can be written as the form $\widetilde{\Gamma}=<A, B, C>$ where $A^{p}=B^{q}=C^{r}=A B C=-1$.

Proposition 5. Let $-1 \in \widetilde{\Gamma}$. Then the classification is listed in the following table. Here $(A, B, C)$ in the third, forth and fifth row are respectively $(1 / 2(1+$ $\dot{\mathrm{i}}+\dot{\mathfrak{j}}+\mathbb{k}), 1 / 2(1+\dot{\mathrm{i}}-\dot{j}+\mathbb{k}), \dot{\mathrm{i}}),(1 / 2(1+\dot{\mathrm{i}}+\dot{j}+\mathbb{k}), 1 / 2(1+\dot{\mathrm{i}}-\dot{\mathfrak{j}}+\mathbb{k}), \dot{\mathrm{i}})$ and $(\cos \pi / 5+\mathbb{k} \cos \pi / 3+\dot{i} \sin \pi / 10, \cos \pi / 3+\mathbb{k} \cos \pi / 5+\dot{j} \sin \pi / 10, \mathbb{k})$. 


\begin{tabular}{|c|c|c|c|c|}
\hline Group, $\widetilde{\Gamma}$ & $\Gamma$ & Order & $(p, q, r)$ & $(A, B, C)$ \\
\hline \hline Cyclic, ${B \mathcal{C}_{n}}$ & $\mathcal{C}_{n}$ & $2 n$ & $(n, n, 1)$ & $\left(e^{\pi \dot{\mathrm{i}} / n}, e^{-\pi \dot{\mathrm{i}} / n},-1\right)$ \\
\hline Binary Dihedron, $B \mathcal{D}_{n}$ & $\mathcal{D}_{n}$ & $4 n$ & $(n, 2,2)$ & $\left(e^{\pi \dot{\mathrm{i}} / n}, \dot{j}, e^{\pi \dot{\mathrm{i}} / n} \dot{\mathfrak{j}}\right)$ \\
\hline Binary Tetrahedron, $B \mathcal{T}$ & $\mathcal{T}$ & 24 & $(3,3,2)$ & $(A, B, C)$ \\
\hline Binary Octahedron, $B \mathcal{O}$ & $\mathcal{O}$ & 48 & $(4,3,2)$ & $(A, B, C)$ \\
\hline Binary Icosahedron, $B \mathcal{I}$ & $\mathcal{I}$ & 160 & $(5,3,2)$ & $(A, B, C)$ \\
\hline
\end{tabular}

The second class: If $\widetilde{\Gamma}$ does not contain -1 , each of its elements $e^{y \alpha}$ represents uniquely the rotation through $2 \alpha$ about $y$, so $\widetilde{\Gamma}$ is isomorphic to a subgroup of $S O(3)$. In this case, none of the rotations can be a half-turn, because then the corresponding quaternion would be pure, and its square would be -1 . Looking through the list of finite subgroups of $S O(3)$, we see that the only kind not containing a half-turn is the cyclic group $\mathcal{C}_{n}$, where $n$ is odd. Hence we have

Proposition 6. The only finite subgroups of quaternion not containing -1 are the cyclic groups of odd order.

Altogether, we obtain the complete classification of the finite subgroups of $S U(2)$.

Theorem 7. (see [6]) Let $\widetilde{\Gamma}$ be a finite subgroup of quaternion.

(1) If $\widetilde{\Gamma}$ does not contain -1 , then $\widetilde{\Gamma}$ is a cyclic subgroup of odd order.

(2) Otherwise, $\widetilde{\Gamma}$ is one of the five types listed in Proposition 5.

2.2. The Outer Automorphisms. By definition,

$$
\operatorname{Inn}(\widetilde{\Gamma})=\left\{A d(x) \mid x \in \widetilde{\Gamma}, A d(x)(y):=x^{-1} y x\right\} .
$$

Slightly different from the standard notations, we denote

$$
\operatorname{Out}(\widetilde{\Gamma})=(\operatorname{Aut}(\widetilde{\Gamma}) \cap \operatorname{Ad}(S U(2))) / \operatorname{Inn}(\widetilde{\Gamma})
$$

and call it the outer automorphism group of $\widetilde{\Gamma}$ by abuse of ambiguity. For $v \in$ $S U(2) \subset \mathbb{H}$, we shall use $O_{v}$ to denote the image of $\operatorname{Ad}(v)$ in $\operatorname{Out}(\widetilde{\Gamma})$ if $\operatorname{Ad}(v) \in$ $\operatorname{Aut}(\widetilde{\Gamma})$. So when $v \in \widetilde{\Gamma}, O_{v}=i d_{\widetilde{\Gamma}}$.

Theorem 8. Up to obvious equivalences, $\left(\widetilde{\Gamma}, O_{v}\right)$ are classified into the following types.

(1) When $O_{v}=i d$, the classification of $\left(\widetilde{\Gamma}, O_{v}\right)$ is the same as that of $\widetilde{\Gamma}$.

(2) Otherwise, there are the following four types.

(i) $\widetilde{\Gamma}=\mathcal{C}_{n}$, and $O_{v}$ is of order 2. When $n=2 m$ is even, that is $\widetilde{\Gamma}=$ $B \mathcal{C}_{m}$, we call $\left(\widetilde{\Gamma}, O_{v}\right)$ of $C_{n}$-type.

(ii) $\widetilde{\Gamma}=B \mathcal{D}_{n}$, and $O_{v}$ is of order $2 .\left(\widetilde{\Gamma}, O_{v}\right)$ is called of $B_{n+1}$-type. 
(iii) $\widetilde{\Gamma}=B \mathcal{T}$, and $O_{v}$ is of order $2 .\left(\widetilde{\Gamma}, O_{v}\right)$ is called of $F_{4}$-type.

(iv) $\widetilde{\Gamma}=B \mathcal{D}_{2}$, and $O_{v}$ is of order $3 .\left(\widetilde{\Gamma}, O_{v}\right)$ is called of $G_{2}$-type.

Proof. This is a direct consequence of the following Proposition 9.

Proposition 9. The outer automorphism group (defined as above) of $\widetilde{\Gamma}$ is $\mathbb{Z}_{2}$ for $\widetilde{\Gamma}=\mathcal{C}_{n}, B \mathcal{D}_{n}(n \neq 2)$ or $B \mathcal{T}$. For $\widetilde{\Gamma}=B \mathcal{D}_{2}$, it is $S_{3}$, the symmetry group of 3 letters. In all other cases, the outer automorphism group is trivial.

Proof. Let $g=e^{\alpha y}=\cos \alpha+y \sin \alpha \in \widetilde{\Gamma}$ and $v=e^{x \beta} \in S U(2)$. Then $\operatorname{Ad}(v)(g)=$ $v^{-1} e^{\alpha y} v=v^{-1}(\cos \alpha) v+v^{-1}(y \sin \alpha) v=\cos \alpha+\left(v^{-1} y v\right) \sin \alpha=e^{\alpha\left(v^{-1} y v\right)}$. Thus we can see that $A d(v)$ transforms the rotation by $2 \alpha$ about the vector $y \in \operatorname{Im}(\mathbb{H})$ into the rotation about $v^{-1} y v \in \operatorname{Im}(\mathbb{H})$ by the same angle. But the action $y \mapsto v^{-1} y v$ itself is the rotation by $2 \beta$ with the vector $x$, since $v=e^{x \beta}$. Then according to the classification of finite subgroups of $S O(3)$ (or $S U(2)$ ), it suffices to show the existence of the non-trivial outer automorphisms $O_{v}$ with $v \in S U(2)$, in the case where $-1 \in \widetilde{\Gamma}$. For convenience, we suppose the generators of $\widetilde{\Gamma}$ are taken as in Remark 5. For $\widetilde{\Gamma}=\mathcal{C}_{n}\left(B \mathcal{D}_{n}(n \neq 2)\right.$ or $B \mathcal{T}$, respectively), we take $v=\dot{\mathfrak{j}}$ (respectively, $\left.e^{-\pi \mathfrak{\mathrm { i }} / 2 n}, \dot{\mathrm{i}} e^{\pi \dot{\mathrm{j}} / 4}\right)$. One can check directly that $v$ satisfies the condition. For $\widetilde{\Gamma}=B \mathcal{D}_{2}$, one can check that $O_{v_{1}}$ and $O_{v_{2}}$ generate the outer automorphism group $S_{3}$, where $v_{1}=e^{(\pi / 3)(\dot{\mathrm{i}}+\mathfrak{j}+\mathbb{k}) / \sqrt{3}}$ and $v_{2}=(\dot{\mathrm{i}}+\mathfrak{j}) / \sqrt{2}$. For the case where $\widetilde{\Gamma}$ does not contain -1 , the result comes from the following lemma.

Lemma 10. Take $\left(\widetilde{\Gamma}, O_{v}\right)$ as above. Let $g \in \widetilde{\Gamma}$ be an element of order $d>1$. If $O_{v}(g)=g^{l}$ then $l \equiv \pm 1 \bmod (d)$. If $g=e^{2 \pi \dot{\mathrm{i}} / d}$, we can take $v=\mathbb{k}$ such that $O_{v}(g)=g^{-1}$.

Proof. Note that $\widetilde{\Gamma}$ must be conjugate with $e^{2 \pi i / d}$ in $S U(2)$. Let $g=u e^{2 \pi i / d} u^{-1}$ with $u \in S U(2)$. Then $h g h^{-1}=g^{l}$ implies that $h u e^{2 \pi \mathrm{i} / d} u^{-1} h^{-1}=u e^{2 \pi \mathfrak{\mathrm { i }} / d} u^{-1}$. Thus $\left(u^{-1} h u\right) e^{2 \pi \dot{\mathrm{i}} / d}\left(u^{-1} h u\right)^{-1}=e^{2 \pi \mathrm{i} k / d}$. Therefore we can assume $g=e^{2 \pi \dot{\mathrm{i}} / d}$. As elements of $S U(2)$,

$$
g=\left(\begin{array}{cc}
e^{2 \pi \dot{\mathrm{i}} / d} & 0 \\
0 & e^{-2 \pi \mathrm{i} / d}
\end{array}\right) \quad \text { and } \quad \mathrm{h}=\left(\begin{array}{cc}
a & b \\
-\bar{b} & \bar{a}
\end{array}\right),
$$

where $a, b \in \mathbb{C}$ with $|a|^{2}+|b|^{2}=1$. One can check that there are only two solutions for $(a, b, l):(1,0,1)$ and $(0,1,-1)$.

Example 11. In the case that $\widetilde{\Gamma}=B \mathcal{D}_{2}$, we can take $\widetilde{\Gamma}=\{ \pm 1, \pm \dot{\mathrm{i}}, \pm \dot{j}, \pm \mathbb{k}\}$, $v=e^{(2 \pi / 3)(\mathfrak{i}+\mathbf{j}+\mathbb{k}) / \sqrt{3}}$ and $A d(v)$ acts just as the permutation $(\dot{\mathrm{i} j} \mathfrak{j} \mathbb{k})$. This is the classical triality. 


\section{McKay Correspondence and Dynkin Diagrams}

In this section we first recall the classical McKay correspondence between subgroups of $S U(2)$ and $A D E$ diagrams [10]. To obtain non-simply laced Dynkin diagrams, one just takes the "folding". In [14], Slodowy considered the relation between finite subgroups of $S U(2)$, Dynkin diagrams and simple singularities, and he realized the diagram automorphisms as the automorphisms of the desingularizations.

By considering the irreducible representations and the conjugacy classes associated with a finite subgroup $\widetilde{\Gamma}$ with an outer automorphism, we give an explicit and unified description for McKay correspondence in all cases.

Let us first recall how to obtain $A D E$-Dynkin diagrams from finite subgroups of $S U(2)$. Let $\widetilde{\Gamma}$ be a finite subgroup of $S U(2)$. Let $W=\mathbb{C}^{2}$ be the standard representation of $S U(2)$. And let $W_{l}, l=0,1, \cdots, s$ be all of the irreducible representations of $\widetilde{\Gamma}$, where $W_{0}$ is the trivial representation, and $s+1$ is the number of the conjugacy classes of $\widetilde{\Gamma}$ (recall that the number of irreducible representations equals to the number of conjugacy classes, for a finite group). Then we have

$$
W \otimes W_{l} \cong \bigoplus_{m} a_{l m} W_{m},
$$

where $a_{l m}$ is the multiplicity of $W_{m}$ in this decomposition. Assigning a node $p_{l}$ to each $W_{l}$ and drawing $a_{l m}$ edges to connect $p_{l}$ and $p_{m}$, we obtain the so-called affine McKay quiver of $\widetilde{\Gamma}$, denoted by $D^{a f f}(\widetilde{\Gamma})$. McKay found that $D^{a f f}(\widetilde{\Gamma})$ was an affine Dynkin diagram of type $A_{n}$ (respectively $\left.D_{n}, E_{6}, E_{7}, E_{8}\right)$ for $\widetilde{\Gamma}=\mathcal{C}_{n+1}$ (respectively $B \mathcal{D}_{n-2}, B \mathcal{T}, B \mathcal{O}, B \mathcal{I}$ ). If we remove the node $p_{0}$ and all the edges with $p_{0}$ as an endpoint, we obtain the corresponding Dynkin diagrams (of finite type), denoted as $D(\widetilde{\Gamma})$.

In the following we consider the pair $\left(\widetilde{\Gamma}, O_{v}\right)$, where $v$ is a unit quaternion.

Definition 12. An irreducible representation of the pair $\left(\widetilde{\Gamma}, O_{v}\right)$ is a representation $W$ of $\Gamma$, which satisfies the following two conditions.

(a) $W$ is invariant under $A d(v)$, that is, $A d(v)$ preserves the characters of $W$.

(b) $W$ can not be written as a direct sum $W_{1} \oplus W_{2}$ with $W_{i}$ satisfying the condition (a).

Definition 13. An $O_{v}$-conjugacy class of $\left(\widetilde{\Gamma}, O_{v}\right)$ is an equivalence class of conjugacy classes of $\widetilde{\Gamma}$, where two conjugacy classes $\bar{g}_{1}$ and $\bar{g}_{2}$ are called equivalent to each other if $\operatorname{Ad}(v)\left(\bar{g}_{1}\right)=\bar{g}_{2}$ or $\operatorname{Ad}(v)\left(\bar{g}_{2}\right)=\bar{g}_{1}$. 
The outer automorphism group $\operatorname{Out}(\widetilde{\Gamma})$ acts on the set of the representations of $\widetilde{\Gamma}$ as follows. Let $O_{v} \in \operatorname{Out}(\widetilde{\Gamma})$, and $V$ be a representation of $\widetilde{\Gamma}$. We obtain a new representation $V^{\prime}$ by composing the action of $\widetilde{\Gamma}$ on $V$ with $O_{v}: \widetilde{\Gamma} \rightarrow \widetilde{\Gamma}$.

Theorem 14. Given any finite subgroup $\widetilde{\Gamma} \subseteq S U(2)$ invariant under $O_{v} \in$ Out $(\widetilde{\Gamma})$, we have

(i) $O_{v}$ induces a diagram automorphism on $D(\widetilde{\Gamma})$;

(ii) the number of irreducible representations of $\left(\widetilde{\Gamma}, O_{v}\right)$ is equal to the number of $O_{v}$-conjugacy classes of $\left(\widetilde{\Gamma}, O_{v}\right)$.

Proof. (ii) is a direct consequence of (i). We check (i) directly in each case.

For $\widetilde{\Gamma}=\mathcal{C}_{n}, \widetilde{\Gamma}$ is generated by $g=e^{2 \pi \mathrm{i} / n}$. For $l=0, \cdots, n-1$, let $W_{l} \cong \mathbb{C}$ be the $l$-th irreducible representation, namely, it is one-dimensional with basis $e_{l}$ satisfying $g\left(e_{l}\right)=g^{l} \cdot e_{l}$. Note that $W_{0}$ is the trivial irreducible representation. Take $v=\dot{j}$. Then $O_{v} \neq i d_{G}$, and $O_{v}(g)=g^{-1}$. So $O_{v}$ transforms the character of $W_{l}$ to the one of $W_{n-l}$. That is, $O_{v}$ induces a diagram automorphism of $D(\widetilde{\Gamma})$.

For $\widetilde{\Gamma}=B \mathcal{D}_{n}$ and $O_{v}$ of order 2 . As a subgroup of $S U(2)$, we can take $\widetilde{\Gamma}$ to be generated by two elements

$$
g=\left(\begin{array}{cc}
e^{\dot{\mathrm{i}} \pi / n} & 0 \\
0 & e^{-\mathrm{i} \pi / n}
\end{array}\right), h=\left(\begin{array}{cc}
0 & 1 \\
-1 & 0
\end{array}\right) .
$$

Let $W_{l} \cong \mathbb{C}^{2}$ be the $l$-th 2 -dimensional representation, with the action given by $g\left(e_{l}, f_{l}\right)=\left(e_{l}, f_{l}\right) g^{l}, h\left(e_{l}, f_{l}\right)=\left((-1)^{l} f_{l}, e_{l}\right)$, where $e_{l}, f_{l}$ is a basis of $W_{l}$. Note that $W_{l}$ is irreducible for $l \neq 0, n$. $W_{0}$ splits into two irreducible representations $W_{01}$ and $W_{02}$, where $W_{02}$ is the trivial one and $\left.g\right|_{W_{01}}=i d,\left.h\right|_{W_{01}}=-i d$. Also $W_{n}$ splits into $W_{n}=W_{n 1} \oplus W_{n 2}$. When $n$ is even, $W_{n 1}=\mathbb{C}<e_{n}+f_{n}>, W_{n 2}=$ $\mathbb{C}<e_{n}-f_{n}>$. When $n$ is odd, $W_{n 1}=\mathbb{C}<e_{n}+\dot{\mathrm{i}} f_{n}>, W_{n 2}=\mathbb{C}<e_{n}-\dot{\mathrm{i}} f_{n}>$. Take $v=\mathbb{k}$, then $O_{v}$ just interchanges the characters of $W_{n 1}$ and $W_{n 2}$.

The situation for $B \mathcal{T}$ is similar but more complicated. The character table for $B \mathcal{T}$ is

\begin{tabular}{|c|c|c|c|c|c|c|c|}
\hline char & 1 & $C$ & -1 & $A^{2}$ & $B^{2}$ & $A$ & $B$ \\
\hline \hline 1 & 1 & 1 & 1 & 1 & 1 & 1 & 1 \\
\hline 2 & 2 & 0 & -2 & -1 & -1 & 1 & 1 \\
\hline 3 & 3 & -1 & 3 & 0 & 0 & 0 & 0 \\
\hline $2^{\prime}$ & 2 & 0 & -2 & $-\rho$ & $-\rho^{2}$ & $\rho^{2}$ & $\rho$ \\
\hline $2^{\prime \prime}$ & 2 & 0 & -2 & $-\rho^{2}$ & $-\rho$ & $\rho$ & $\rho^{2}$ \\
\hline $1^{\prime}$ & 1 & 1 & 1 & $\rho$ & $\rho^{2}$ & $\rho^{2}$ & $\rho$ \\
\hline $1^{\prime \prime}$ & 1 & 1 & 1 & $\rho^{2}$ & $\rho$ & $\rho$ & $\rho^{2}$ \\
\hline
\end{tabular}


where $\rho=e^{2 \pi i / 3}$, and the entries in the first column are all irreducible representations indexed by the affine Dynkin diagram of $E_{6}$. Let $O_{v}$ interchange the generators $A$ and $B$. Then $O_{v}$ interchanges pairwise the 5 -th and the 6 -th columns, the 7-th and the 8-th columns in this table. Hence it also interchanges pairwise the 5 -th and the 6 -th rows, the 7 -th and the 8 -th rows. That is, $O_{v}$ induces a diagram automorphism of $D(\widetilde{\Gamma})$.

The proof for $G=B \mathcal{D}_{2}$ is very easy, according to Remark 11 .

Similar to the classical case, we define the McKay quiver for a pair $\left(\widetilde{\Gamma}, O_{v}\right)$ as follows.

Definition 15. Given a pair $\left(\widetilde{\Gamma}, O_{v}\right)$, let $W_{l}, l=0, \cdots, n$ be all irreducible representations of $\left(\widetilde{\Gamma}, O_{v}\right)$, where $W_{0}$ is the trivial one, and $n+1$ is the number of the $O_{v}$-conjugacy classes. Assign a node $p_{l}$ to each irreducible representation $W_{l}$. Let $W=\mathbb{C}^{2}$ be the standard representation of $S U(2)$. Assume that $W_{l} \otimes W \cong \oplus_{m} a_{l m} W_{m}$. When $a_{l m}=a_{m l} \neq 0$ (it must be equal to 1 in this situation), we draw $a_{l m}$ edges to connect the nodes $p_{l}$ and $p_{m}$. When $a_{l m} \neq a_{m l}$, one of them, say $a_{l m}$, must be 2 or 3 , and another is 1 . In this case, we draw $a_{l m}$ directed edges from $p_{m}$ to $p_{l}$. Thus, we obtain a diagram, called the affine McKay quiver of $\left(\widetilde{\Gamma}, O_{v}\right)$, and denoted by $D_{\left.\widetilde{\Gamma}, O_{v}\right)}^{a f f}$. Removing the node $p_{0}$ and all the edges with $p_{0}$ as an endpoint from $D_{\left(\widetilde{\Gamma}, O_{v}\right)}^{a f f}$, we obtain a diagram, denoted by $D_{\left(\widetilde{\Gamma}, O_{v}\right)}$, called the (finite) McKay quiver of $\left(\widetilde{\Gamma}, O_{v}\right)$.

Theorem 16. Given any finite subgroup $\widetilde{\Gamma} \subseteq S U(2)$ and $O_{v} \in \operatorname{Out}(\widetilde{\Gamma}), D_{\left(\widetilde{\Gamma}, O_{v}\right)}$ must be one of the following types.

(1) For $O_{v}=i d_{\widetilde{\Gamma}}, D_{\left(\widetilde{\Gamma}, O_{v}\right)}$ is a Dynkin diagram of ADE-type.

(2) Suppose $O_{v}$ is non-trivial.

(i) For $\widetilde{\Gamma}=\mathcal{C}_{2 n+1}, D_{\left(\widetilde{\Gamma}, O_{v}\right)}$ is of $A_{n}$-type.

(ii) For $\widetilde{\Gamma}=B \mathcal{C}_{n}=\mathcal{C}_{2 n}, D_{\left(\widetilde{\Gamma}, O_{v}\right)}$ is of $C_{n}$-type.

(iii) For $\widetilde{\Gamma}=B \mathcal{D}_{n}$ and $\left(O_{v}\right)^{2}=i d_{\widetilde{\Gamma}}, D_{\left(\widetilde{\Gamma}, O_{v}\right)}$ is of $B_{n+1}$-type.

(iv) For $\widetilde{\Gamma}=B \mathcal{T}, D_{\left(\widetilde{\Gamma}, O_{v}\right)}$ is of $F_{4}$-type.

(v) For $\widetilde{\Gamma}=B \mathcal{D}_{2}$ and $\left(O_{v}\right)^{3}=i d_{\widetilde{\Gamma}}, D_{\left(\widetilde{\Gamma}, O_{v}\right)}$ is of $G_{2}$-type.

Proof. (1) is the classical McKay correspondence, see [10]. By Theorem 14, we obtain non-simply laced Dynkin diagrams from simply laced ones. Let $\left\{W_{l} \mid l=\right.$ $0,1, \cdots, s\}$ is the set of all irreducible representations of $\left(\widetilde{\Gamma}, O_{v}\right)$, where $s+1$ is the number of the $O_{v}$-conjugacy classes of $\left(\widetilde{\Gamma}, O_{v}\right)$, and $W_{0}$ is the trivial one. Let $a_{l m}, l, m=0, \cdots, s$ be the non-negative numbers determined by the following 
decomposition

$$
W_{l} \otimes W \cong \bigoplus_{m} a_{l m} W_{m}
$$

We only need to verify the number of undirected edges connecting the nodes $p_{l}$ and $p_{m}$ is equal to $a_{l m}$ where $a_{l m}=a_{m l}$; and the number of directed edges connecting from $p_{m}$ to $p_{l}$ is equal to $a_{l m}$ where $a_{l m}>a_{m l}=1$.

This follows from a direct checking. For example, we compute $D_{\left(\widetilde{\Gamma}, O_{v}\right)}$ for $\widetilde{\Gamma}=\mathcal{C}_{2 k}$ and $O_{v} \neq i d$. Just as in the proof of Theorem 14, suppose $\widetilde{\Gamma}$ is generated by $g=e^{2 \pi \dot{\mathrm{i}} /(2 k)}$. Let $W_{l} \cong \mathbb{C}$ is the $l$-th irreducible representation of $\widetilde{\Gamma}$, which is one-dimensional with basis $e_{l}$, where $g\left(e_{l}\right)=g^{l} \cdot e_{l}$, for $l=0, \cdots, 2 k-1$. Note that $W_{0}$ is the trivial irreducible representation. Take $v=\dot{j}$. Then $O_{v} \neq i d_{\widetilde{\Gamma}}$. And $O_{v}(g)=g^{-1}$. So the irreducible representations for $\left(\widetilde{\Gamma}, O_{v}\right)$ are $U_{0}=W_{0}, U_{l}=$ $W_{l} \oplus W_{2 k-l}, 0<l<k, U_{k}=W_{k}$. Let $U_{l}=\bigoplus a_{l m} U_{m}$. Then we can see that $a_{l m}$ satisfies the constrains, since $U_{0} \otimes W=U_{1}^{m}, U_{1} \otimes W=2 U_{0} \oplus U_{2}, U_{l} \otimes W=$ $U_{l-1} \oplus U_{l+1}$, for $1<l<k-2$ and $U_{k-1} \otimes W=U_{k-2} \oplus 2 U_{k}, U_{k} \otimes W=U_{k-1}$.

Corollary 17. Except the case $\left(\widetilde{\Gamma}, O_{v}\right)$ where $\widetilde{\Gamma}=\mathcal{C}_{2 k+1}$ and $O_{v} \neq i d_{\widetilde{\Gamma}}, D_{\left(\widetilde{\Gamma}, O_{v}\right)}$ is just one of the affine Dynkin diagrams of untwisted type, and the matrix $2 I-\left(a_{i j}\right)$ is just the Cartan matrix of the corresponding affine Dynkin diagram.

Remark 18. In fact, this outer automorphism $O_{v}$ induces an outer automorphism of a regular polyhedron $\mathcal{P}$ (that is, interchanging the vertices and the faces of $\mathcal{P}$ ). For example, the tetrahedron has such a non-trivial outer automorphism. Accordingly the binary tetrahedron group as well as its McKay quiver also has such an outer automorphism.

\section{Triality and Non-Simply Laced McKay Correspondence}

We have seen that the McKay correspondence in non-simply laced cases is induced by that one in simply laced case with a symmetry of $\mathbb{H}$. Essentially, all symmetries of $\mathbb{H}$ come from the triality property on $\mathbb{H}$. In this section, we formulate this correspondence in terms of triality.

4.1. Triality. In the following, we recall the theory on triality. For references, see [1] and [2]. Let $V_{i}, i=1,2,3$ be three real vector spaces of finite dimension.

Definition 19. A triality is a trilinear map

$$
t: V_{1} \otimes V_{2} \otimes V_{3} \rightarrow \mathbb{R}
$$

such that for any non-zero $v_{1} \in V_{1}, v_{2} \in V_{2}$ there exists a $v_{3} \in V_{3}$ such that $t\left(v_{1} \otimes v_{2} \otimes v_{3}\right) \neq 0$ (and similarly for $v_{1}, v_{3} \neq 0, v_{2}, v_{3} \neq 0$ ). If each $V_{i}$ has a norm, we say that $f$ is a normed triality if $\left|t\left(v_{1} \otimes v_{2} \otimes v_{3}\right)\right| \leq\left\|v_{1}\right\| \cdot\left\|v_{2}\right\| \cdot\left\|v_{3}\right\|$, 
and for all $v_{1}, v_{2} \neq 0$, there is a $v_{3} \neq 0$ for which the bound is attained (and similarly for the other two cases).

Example 20. $V_{1}=V_{2}=V_{3}=\mathbb{R}, \mathbb{C}$, or $\mathbb{H}$ respectively and take $t(x \otimes y \otimes z)=$ $\operatorname{Re}(x y z)$. Then $t$ is a normed triality.

Theorem 21. A triality exists only if $\operatorname{dim} V_{1}=\operatorname{dim} V_{2}=\operatorname{dim} V_{3}=1,2,4$ or 8 .

Proof. See [1] for reference. For later uses, we sketch the proof. Suppose we are given a triality $t$. Then for any $e_{1} \neq 0$ we get a duality of $V_{2}, V_{3}$, so we must have $\operatorname{dim} V_{2}=\operatorname{dim} V_{3}$ and similarly $\operatorname{dim} V_{1}=\operatorname{dim} V_{2}=\operatorname{dim} V_{3}$. We can transpose $V_{3}$ to get $t^{\prime}: V_{1} \otimes V_{2} \rightarrow V_{3}^{*}$. Choose $e_{1} \neq 0$ in $V_{1}$ and use it to identify $V_{2}$ with $V_{3}^{*}$ by $v_{2} \mapsto t^{\prime}\left(e_{1} \otimes v_{2}\right)$. Similarly choose $e_{2} \neq 0$ in $V_{2}$ to identify $V_{1}$ with $V_{3}^{*}$. We now have $t^{\prime \prime}: V_{3}^{*} \otimes V_{3}^{*} \rightarrow V_{3}^{*}$ for which $t^{\prime \prime}\left(e_{1} \otimes e_{2}\right)$ acts as a two-sided unit and $t^{\prime \prime}$ is non-singular in that if $x, y \neq 0 \in V_{3}^{*}$, then $t^{\prime \prime}(x \otimes y) \neq 0$. So we have a division algebra $\mathbb{A}$ over $\mathbb{R}$ and consequently $\operatorname{dim} V_{i}=1,2,4$ or 8 . If we start with a normed triality, we then obtain a normed algebra $\mathbb{A}$, and in this case, we should take unit vectors $e_{1}, e_{2}$ and $e_{3}$.

From the above proof we see that for a normed triality $t: V_{1} \times V_{2} \times V_{3} \rightarrow \mathbb{R}$, given a triple $\vec{v}=\left(v_{1}, v_{2}, v_{3}\right)$ where $v_{i} \in V_{i}, i=1,2,3$ such that $\left\|v_{i}\right\|=1$ and $t\left(v_{1}, v_{2}, v_{3}\right)=1$, we obtain a normed division algebra $\mathbb{A}$. Such a triple $\vec{v}=\left(v_{1}, v_{2}, v_{3}\right)$ is called a triality triple. We will write $t_{\mathbb{A}}$ instead of $t$ and denote the set of all triality triples as $T r i_{\mathbb{A}}$, that is

$$
\operatorname{Tr}_{\mathbb{A}}=\left\{\left(v_{1}, v_{2}, v_{3}\right) \in V_{1} \times V_{2} \times V_{3} \mid t\left(v_{1}, v_{2}, v_{3}\right)=1,\left\|v_{i}\right\|=1, i=1,2,3\right\} .
$$

It is well-known that there are only four normed (finite) $\mathbb{R}$-algebras and they are $\mathbb{R}, \mathbb{C}, \mathbb{H}, \mathbb{O}$.

An automorphism of the normed triality $t: V_{1} \times V_{2} \times V_{3} \rightarrow \mathbb{R}$ is a triple of norm-preserving maps $f_{i}: V_{i} \rightarrow V_{i}$ such that

$$
t(f(\vec{v}))=t(\vec{v})
$$

for all $\vec{v} \in V_{1} \times V_{2} \times V_{3}$, where $f=\left(f_{1}, f_{2}, f_{3}\right), \vec{v}=\left(v_{1}, v_{2}, v_{3}\right)$ and $f(\vec{v})=$ $\left(f_{1}\left(v_{1}\right), f_{2}\left(v_{2}\right), f_{3}\left(v_{3}\right)\right)$. These automorphisms form a group we call $A u t\left(t_{\mathbb{A}}\right)$. Take a triality triple $\vec{v}=\left(v_{1}, v_{2}, v_{3}\right) \in T r i_{\mathbb{A}}$, and denote

$$
\operatorname{Aut}\left(t_{\mathbb{A}}, \vec{v}\right)=\left\{f \in \operatorname{Aut}\left(t_{\mathbb{A}}\right): f(\vec{v})=\vec{v}\right\} .
$$

Lemma 22. Aut $\left(t_{\mathbb{A}}\right)$ acts transitively on the set Tri $i_{\mathbb{A}}$ of triality triples, and there is a canonical isomorphism $\psi_{\vec{v}}:$ Aut $(\mathbb{A}) \stackrel{\sim}{\longrightarrow}$ Aut $\left(t_{\mathbb{A}}, \vec{v}\right)$ for any triality triple $\vec{v}$.

Proof. The first part is trivial since given two unit vectors $u_{i}, v_{i}$ in $V_{i}$, there is a norm-preserving map $f_{i} \in \operatorname{Aut}\left(V_{i}\right)$, such that $f_{i}\left(u_{i}\right)=v_{i}$. To prove the second part, we identify $V_{i}$ to $\mathbb{A}$ by identifying $v_{i}$ to the identity element 1 of $\mathbb{A}$. Then for 
any $f \in \operatorname{Aut}\left(t_{\mathbb{A}}, \vec{v}\right)$, under above identifications, $f$ preserves the algebra structure of $\mathbb{A}$, therefore $f \in A u t(\mathbb{A})$. The converse is also true.

Corollary 23. For all $\vec{u}, \vec{v} \in T r i_{\mathbb{A}}$, there exists a $\phi_{\vec{u} \vec{v}} \in \operatorname{Aut}\left(t_{\mathbb{A}}\right)$, such that $\operatorname{Aut}\left(t_{\mathbb{A}}, \vec{u}\right)=\phi_{\vec{u} \vec{v}}^{-1}\left(A u t\left(t_{\mathbb{A}}, \vec{v}\right)\right) \phi_{\vec{u} \vec{v}}$.

Let $p_{i}: A u t\left(t_{\mathbb{A}}\right) \rightarrow O\left(V_{i}\right)$ be the homomorphism induced by the projection to the $i^{\text {th }}$ component: $V_{1} \times V_{2} \times V_{3} \rightarrow V_{i}, i=1,2,3$. Then by construction, for any $\vec{v} \in \operatorname{Tri}_{\mathbb{A}}$, the map $p_{i}: \operatorname{Aut}\left(t_{\mathbb{A}}, \vec{v}\right) \stackrel{\sim}{\longrightarrow} p_{i}\left(\operatorname{Aut}\left(t_{\mathbb{A}}, \vec{v}\right)\right) \subset O\left(V_{i}\right)$ is an isomorphism onto its image, for all $i$.

4.2. McKay Correspondence via Triality of $\mathbb{H}$. From now on, we assume $\mathbb{A}=\mathbb{H}$. The universal cover (i.e. double cover) $\widetilde{\operatorname{Aut}}\left(t_{\mathbb{H}}\right)$ of $\operatorname{Aut}\left(t_{\mathbb{H}}\right)$ is isomorphic to $S U(2)^{3}$. Let $\widetilde{\Gamma}$ be a finite subgroup of $\widetilde{A u t}\left(t_{\mathbb{H}}\right)$ with the image $\Gamma \subset A u t\left(t_{\mathbb{H}}\right)$. A subgroup of $\widetilde{A u t}\left(t_{\mathbb{H}}\right)$ is called an isotropic subgroup if it fixes some element in $T r i_{\mathbb{H}}$. By Lemma 22, a finite isotropic subgroup of $\widehat{A u t}\left(t_{\mathbb{H}}\right)$ is in fact a subgroup of $\overline{A u t}(\mathbb{H}) \cong S U(2)$. Thus finite subgroups of $S O(3)$ (or $S U(2))$ are identified with finite isotropic subgroups of $A u t\left(t_{\mathbb{H}}\right)$ (or $\widetilde{A u t}\left(t_{\mathbb{H}}\right)$ ).

Considering the pairs of finite isotropic subgroups $\widetilde{\Gamma}, \widetilde{\Gamma^{\prime}}$ of $\widetilde{A u t}\left(t_{\mathbb{H}}\right)$, of the same order, with $p_{1}(\Gamma)=p_{1}\left(\Gamma^{\prime}\right)$ (or equivalently, for all $p_{i}$ ). In the following we show that the classification of such pairs is equivalent to the classification of the pairs $\left(\widetilde{\Gamma}, O_{v}\right)$, where $\widetilde{\Gamma} \subset S U(2), v \in \mathbb{H}$.

Assume $\vec{u}, \vec{v} \in T r i_{\mathbb{H}}$. According to [1], we have

$$
S O(3)=\operatorname{Aut}(\mathbb{H}) \cong A u t\left(t_{\mathbb{H}}, \vec{u}\right) \subset A u t\left(t_{\mathbb{H}}\right) \cong(S p(1) \times S p(1) \times S p(1)) /\{ \pm 1\} .
$$

Without loss of generality, we can take $\vec{u}=\vec{e}=(1,1,1)$ and $\vec{v}=\left(v_{1}, v_{2}, v_{3}\right)$. In this case, we can take $V_{i}=\mathbb{H}, i=1,2,3$, then the map $t_{\mathbb{H}}: \mathbb{H} \times \mathbb{H} \times \mathbb{H} \rightarrow$ $\mathbb{R}$ given by $t(u, v, w)=R e(u v w)$ defines the normed triality of $\mathbb{H}$. And this triality implies that any triality triple $\left(v_{1}, v_{2}, v_{3}\right)$ determines unique isomorphisms $V_{1} \cong V_{2} \cong V_{3}=\mathbb{H}$ (every isomorphism is in fact an automorphism of $\mathbb{H}$ ). And $S O(3)=\operatorname{Aut}\left(t_{\mathbb{H}}, \vec{u}\right) \cong A u t\left(t_{\mathbb{H}}, \vec{v}\right)$, where the isomorphism, is just $\phi_{\vec{v}}:=\phi_{\vec{u} \vec{v}}$ as above, is given by $g \mapsto\left(\phi_{\vec{v}, 1}(g), \phi_{\vec{v}, 2}(g), g\right)$ with $\phi_{\vec{v}, i}$ being an isomorphism of groups induced by $\vec{v}=\left(v_{1}, v_{2}, v_{3}\right)$. Note that the dual space $\mathbb{H}^{*}$ is naturally identified with $\overline{\mathbb{H}}=\mathbb{H}$, where $\bar{x}=\operatorname{Re}(x)-\operatorname{Im}(x)$, since the inner product on $\mathbb{H}$ is defined as

$$
<x, y>:=\operatorname{Re}(\bar{x} y)=\operatorname{Re}(x \bar{y}) .
$$

This implies for $v_{1} \in V_{1}, t$ induces an isomorphism from $V_{2}=\mathbb{H}$ to $\mathbb{H}$ defined by $t_{1}: y \mapsto v_{1} y$ for $y \in V_{2}$. Thus $\phi_{\vec{v}, 1}(g)=t_{1}^{-1} g t_{1}$. Similarly $\phi_{\vec{v}, 2}(g)=t_{2}^{-1} g t_{2}$ with $t_{2}$ defined by $t_{2}(x)=x v_{2}$ for $x \in V_{1}$. Since $v_{1}, v_{2}$ are unit quaternions, $\phi_{\vec{v}, 1}, \phi_{\vec{v}, 2}$ are elements of $S U(2)$. Pulled back to $S U(2), \phi_{\vec{v}, 1}, \phi_{\vec{v}, 2}$ induces automorphisms of $S U(2)$, which are just the conjugations $A d\left(v_{1}\right): g \mapsto v_{1}^{-1} g v_{1}$ and the identity. 
The reason is the following. Let $g \in S U(2)$, then $g(x)=g \cdot x$, since we consider $S U(2)$ as the group of unit quaternions. We have the following commutative diagram:

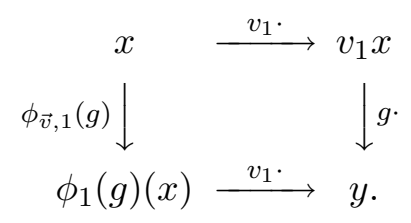

Thus $\phi_{\vec{v}, 1}(g)=\operatorname{Ad}\left(v_{1}\right)(g)=v_{1}^{-1} g v_{1}$. Similarly, we have $\phi_{\vec{v}, 2}(g)=g$. Hence the isomorphism $\phi_{\vec{v}}=\left(A d\left(v_{1}\right), i d, i d\right)$. Thus we have proved that

Proposition 24. Let $\widetilde{\Gamma}, \widetilde{\Gamma^{\prime}}$ be two finite subgroups of $\widetilde{A u t}\left(t_{\mathbb{H}}\right)$ of the same order, fixing respectively $\vec{u}, \vec{v} \in T r i_{\mathbb{H}}$, and let $p_{1}(\widetilde{\Gamma})=p_{1}\left(\widetilde{\Gamma^{\prime}}\right)$. Then $\phi_{\vec{u} \vec{v}}$ induces an (outer) automorphism on $p_{1}(\widetilde{\Gamma})=p_{1}\left(\widetilde{\Gamma^{\prime}}\right)$.

Let $\widetilde{\Gamma} \subset S U(2)$, and $\widetilde{\Gamma^{\prime}}, \widetilde{\Gamma^{\prime \prime}}$ be two finite isotropic subgroups of $\widetilde{A u t}\left(t_{\mathbb{H}}\right)$, of the same order, and assume $p_{1}\left(\widetilde{\Gamma^{\prime}}\right)=p_{1}\left(\widetilde{\Gamma^{\prime \prime}}\right)$. Let $v \in \mathbb{H}$. Consider the equivalence classes of the pairs $\left(\widetilde{\Gamma^{\prime}}, \widetilde{\Gamma^{\prime \prime}}\right)$ in the obvious sense.

Theorem 25. The equivalence classes of the pairs $\left(\widetilde{\Gamma^{\prime}}, \widetilde{\Gamma^{\prime \prime}}\right)$ as above are in oneto-one correspondence with the equivalence classes of the pairs $\left(\widetilde{\Gamma}, O_{v}\right)$.

Proof. Given a pair $\left(\widetilde{\Gamma^{\prime}}, \widetilde{\Gamma^{\prime \prime}}\right)$ as above, up to conjugation, we can take $\vec{u}=(1,1,1)$ and $\vec{v}=\left(v_{1}, v_{2}, v_{3}\right)$. We take $\widetilde{\Gamma} \subset S U(2)$ to be the finite subgroup of the same order such that $\Gamma=p_{1}\left(\widetilde{\Gamma^{\prime}}\right)=p_{1}\left(\widetilde{\Gamma^{\prime \prime}}\right)$, and we take $x=v_{1}$. Conversely, given $\left(\widetilde{\Gamma}, O_{x}\right)$, we take $\vec{u}=(1,1,1)$ and $\vec{v}=\left(x, x^{-1}, 1\right)$. Then we can take $\widetilde{\Gamma^{\prime \prime}}$ to be group consisting of the diagonal elements of $\widetilde{\Gamma} \times \widetilde{\Gamma} \times \widetilde{\Gamma}$, and $\widetilde{\Gamma}^{\prime}=(A d(x), i d, i d)\left(\widetilde{\Gamma^{\prime \prime}}\right)$. Then by construction and by Proposition 24, one can easily see the above correspondence is one-to-one.

Corollary 26. The equivalence classes of the pairs $\left(\widetilde{\Gamma^{\prime}}, \widetilde{\Gamma^{\prime \prime}}\right)$ as in Proposition 24, are in one-to-one correspondence with the pairs $(D, \tau)$ with $D$ a simply laced Dynkin diagram and $\tau$ a diagram automorphism (see Figure 1).

Remark 27. The same arguments as the proof of Proposition 24 and Theorem 25 work in fact for all normed division algebras.

Much less is known about general finite subgroups of $G_{2}=\operatorname{Aut}(\mathbb{O})$. For finite subgroups of $S U(3) \subset G_{2}$, there is a geometric McKay correspondence ([12][13] etc). But the McKay quiver is usually more complicated. We hope to have a detailed study of the octonion case in the future. 


\section{REFERENCES}

[1] J.F. Adams, Lectures on Exceptional Lie Groups, The University of Chicago Press, 1996.

[2] J.C. Baez, The octonions, Bull. Amer. Math. Soc. 39 (2002), 145-205.

[3] T. Bridgeland, A. King, M. Reid, The McKay correspondence as an equivalence of derived categories, J. Amer. Math. Soc. 14 (2001), no. 3, 535-554.

[4] E. Brieskorn, Rationale singularitäten komplexer flächen (German), Invent. Math. 4, 1967/1968, 336-358.

[5] H.S.M. Coxeter, Regular polytopes, Second edition, The Macmillan Co., New York; CollierMacmillan Ltd., London 1963.

[6] H.S.M. Coxeter, Regular complex polytopes, Second edition, Cambridge University Press, Cambridge, 1991.

[7] P. Du Val, Homographies quaternions and rotations, Oxford Mathematical Monographs Clarendon Press, Oxford, 1964.

[8] Y.-H. He, Closed String Tachyons, Non-Supersymmetric Orbifolds and Generalised McKay Correspondence, Adv. Theor. Math. Phys. 7(2003), 121-144.

[9] J.E. Humphreys, Introduction to Lie Algebras and Representation Theory, Springer-Verlag, New York-Berlin, 1978.

[10] J. McKay, Graphs, singularities and finite groups, Proc. Symp. in Pure Math, 37, 1980, 183-186.

[11] H. Pinkham, Séminaire sur les Singularités des Surfaces, LNM 777, Springer, Berlin, 1980.

[12] M. Reid, La correspondance de McKay [The McKay correspondence], Séminaire Bourbaki, Vol. 1999/2000, Astérisque No. 276 (2002), 53-72.

[13] S.S. Roan, Minimal resolutions of Gorenstein orbifolds in dimension three, Topology 35 (1996), no. 2, 489-508.

[14] P. Slodowy, Simple Singularities and simple algebraic groups, LNM 815, Springer, Berlin, 1980.

[15] J.S. Song, Three-Dimensional Gorenstein Singularities and SU(3) Modular Invariants, arXiv:hep-th/9908008.

[16] C. Vafa, Mirror symmetry and closed string tachyon condensation, arXiv:hep-th/0111051.

Naichung Conan Leung

the Institute of Mathematical Sciences and Department of Mathematics

The Chinese University of Hong Kong

Shatin, N.T., Hong Kong.

E-mail: leung@ims.cuhk.edu.hk

Jiajin Zhang

Department of Mathematics,

Sichuan University,

Chengdu, 610065, P.R. China

E-mail: jjzhang@scu.edu.cn 